\title{
Estudo cinético da etapa de hidrólise enzimática da palha da cana- de-açúcar: efeito da velocidade de agitação e da concentração de substrato
}

\author{
B. PRATTO ${ }^{1}$, R. B. A. SOUZA ${ }^{1}$, R. SOUSA Jr ${ }^{1}$, A. J. G. CRUZ ${ }^{1}$ \\ ${ }^{1}$ Universidade Federal de São Carlos, PPGEQ-UFSCar, Programa de Pós-Graduação em \\ Engenharia Química \\ E-mail: brunapratto@hotmail.com
}

\begin{abstract}
RESUMO - Entre os diferentes tipos de biomassa lignocelulósica, a palha de cana-de-açúcar tem se destacado como fonte de matéria-prima para a produção de etanol 2G. No entanto, um dos desafios que envolvem a sua produção é maximizar a conversão de celulose em açúcares fermentescíveis na etapa de hidrólise. Uma das formas de melhorar esse processo é minimizar os efeitos difusionais externos, de forma a garantir que o processo não seja limitado por essa etapa. O objetivo deste trabalho foi realizar estudo cinético da hidrólise da celulose, avaliando o efeito da velocidade de agitação e da concentração de substrato. Experimentos empregando palha de cana-de-açúcar, pré-tratada hidrotermicamente $\left(195^{\circ} \mathrm{C} / 10 \mathrm{~min}, 200 \mathrm{rpm}, 1: 10 \mathrm{~s}-1\right)$, foram realizados em frascos agitados $\left(50^{\circ} \mathrm{C}, \mathrm{pH} \mathrm{5,5} \mathrm{FPU} \cdot \mathrm{g}_{\text {celulose }}{ }^{-1}, 10 \%\right.$ de sólidos $\left.\mathrm{m} / \mathrm{v}\right)$ em 0,50 , 150, 200, 250 e $300 \mathrm{rpm}$. Para agitações acima de $200 \mathrm{rpm}$, as velocidades iniciais de reação foram praticamente invariantes, obtendo um valor médio de $0,052 \mathrm{~g}_{\text {glicose }} \mathrm{L}^{-1} \cdot \mathrm{min}^{-1}$. Após determinada a condição ótima de agitação, foram realizados experimentos com cargas de sólidos de 2,5, 5, 7,5 e $10 \%(\mathrm{~m} / \mathrm{v})$, mantendo-se constantes as variáveis operacionais: $50^{\circ} \mathrm{C}, \mathrm{pH} 5,284 \mathrm{FPU} \cdot \mathrm{L}^{-1}$ e $200 \mathrm{rpm}$. Assim, foi possível ajustar ao modelo pseudo homogêneo de Michaelis-Menten, obtendo $V_{\text {máx }}=0,059 \mathrm{~g}_{\text {glicose }} \cdot \mathrm{L}^{-1} \cdot \mathrm{min}^{-1}$ e $\mathrm{K}_{\mathrm{m}}=15,339 \mathrm{~g}_{\text {glicose }}$ potencial $\mathrm{L}^{-1}$. A concentração de glicose foi o parâmetro utilizado como resposta, a fim de avaliar as velocidades iniciais de reação.
\end{abstract}

\section{INTRODUÇÃO}

É crescente a demanda mundial por combustíveis, seja para transporte, atividades industriais ou geração de energia elétrica. De acordo com a Agência Internacional de Energia (AIE), os combustíveis fósseis continuam a satisfazer a maior parte das demandas energéticas mundiais, no entanto, essa dependência dos combustíveis não renováveis está ameaçando o clima e os ecossistemas da Terra. A fim de minimizar os impactos ambientais gerados pelo uso de combustíveis fósseis, têm se tornado cada vez mais intensas as pesquisas em busca de novas energias renováveis. Nesse cenário, os resíduos lignocelulósicos apresentam-se como uma fonte renovável muito promissora e de grande potencial para a produção de etanol (KOOTSTRA et al., 2009; SARKAR et al., 2012).

Dentre os resíduos agroindustriais existentes no Brasil, a palha de cana-de-açúcar está em uma posição de destaque, devido à grande quantidade disponível no campo e por 
representar, aproximadamente, um terço da energia total da cana-de-açúcar (LEAL et al., 2013).

Embora as biomassas lignocelulósicas representem uma fonte atrativa para a produção de etanol de segunda geração, um dos principais desafios envolvidos é obter altas taxas de conversões de polissacarídeos em açúcares fermentescíveis, na etapa de hidrólise (SANTOS et al., 2012). Na hidrólise enzimática, vários são os fatores que influenciam o rendimento dessa etapa, tais como: propriedades físicas do substrato, tipo de pré-tratamento empregado, ação sinergética das enzimas, agitação do meio reacional, temperatura, $\mathrm{pH}$, concentração de enzima e de substrato (BINOD et al., 2011; MAEDA et al., 2011). Esse rendimento está associado à velocidade de formação de glicose, que é governada por três eventos em sequência: velocidade de transferência de massa da enzima até a superfície do substrato, velocidade de adsorção da enzima ao substrato e velocidade da reação enzimática (GAN et al., 2003). Sendo assim, uma das maneiras de melhorar o processo de sacarificação enzimática é minimizar os efeitos de resistência à transferência de massa, de forma a garantir que o processo não seja limitado por essa etapa.

Dessa maneira, o principal objetivo desse trabalho foi estudar a cinética da hidrólise enzimática da palha da cana-de-açúcar, avaliando o efeito da velocidade de agitação e da concentração de substrato na velocidade inicial de reação.

\section{MATERIAIS E MÉTODOS}

\subsection{Materiais}

A palha de cana-de-açúcar utilizada neste trabalho foi fornecida pela Usina Itapira, localizada em Catanduva, SP.

Para a realização dos experimentos de hidrólise enzimática, utilizou-se o complexo enzimático Cellic ${ }^{\circledR} \mathrm{CTec} 2$, doado pela Novozymes Latin America (Araucária, PR).

\subsection{Métodos}

Caracterização química da palha de cana-de-açúcar: Amostras de palha in natura e prétratada foram caracterizadas quanto a sua composição química de acordo com a metodologia de Rocha et al. (1997), validada por Gouveia et al. (2009).

Pré-tratamento hidrotérmico: Para a aplicação do pré-tratamento hidrotérmico foi utilizado um reator (modelo 4532, Parr Instrument Company, Moline, Illinois, EUA). Antes de iniciar o pré-tratamento, a palha de cana-de-açúcar in-natura foi moída a 5 mesh e determinada a sua umidade. Em seguida, foi adicionada ao reator e misturada com água na proporção 1:10 (massa sólido/massa líquido), sendo submetida a $195^{\circ} \mathrm{C} / 10$ minutos e 200 rpm. Ao final da reação a fração sólida foi separada por filtração e lavada com água para remoção dos componentes solubilizados. Depois de realizado o pré-tratamento, coletaram-se amostras da palha pré-tratada para a caracterização química. 
Hidrólise enzimática: Os experimentos de hidrólise enzimática foram realizados em frascos de Erlenmeyers de $250 \mathrm{~mL}$, com volume total de reação de $50 \mathrm{~mL}$, colocados em shaker (modelo 832, Marconi) nas seguintes condições operacionais: temperatura de $50^{\circ} \mathrm{C}$, pH 5 (tampão citrato de sódio $50 \mathrm{mM}$ ), concentração de substrato de $10 \%$ de sólidos (massa de palha/volume reacional) e concentração de enzima de $5 \mathrm{FPU} \cdot \mathrm{g}_{\text {celulose }}{ }^{-1}$, variando a agitação em $0,50,150,200,250$ e $300 \mathrm{rpm}$.

Para determinar o efeito da concentração de substrato, experimentos foram realizados em erlenmeyers de $250 \mathrm{~mL}$, variando a concentração de sólidos de 2,5; 5; 7,5 e $10 \%$ (massa de palha seca/volume reacional). Em cada batelada experimental realizada em shaker (modelo 832, Marconi), utilizou-se um volume total de reação de $50 \mathrm{~mL}$. Manteve-se constante a temperatura em $50^{\circ} \mathrm{C}$, considerando agitação de $200 \mathrm{rpm}$ e concentração volumétrica de atividade enzimática da Cellic ${ }^{\circledR} \mathrm{CTec} 2$ de $284 \mathrm{FPU} \cdot \mathrm{L}_{\text {solução }}{ }^{-1}$, em tampão citrato de sódio 50 $\mathrm{mM}$ a $\mathrm{pH} 5$.

Durante cada ensaio experimental foram retiradas alíquotas armazenadas em $\mathrm{NaOH} \mathrm{0,2}$ M, na proporção $300 \mu \mathrm{L} \mathrm{NaOH}: 500 \mu \mathrm{L}$ amostra, para inativação da enzima. As alíquotas foram retiradas nos tempos de reação 5, 10, 15, 30, 60, 120 e 240 minutos. Todos os ensaios foram realizados em triplicata.

Quantificação de glicose: Para a quantificação de glicose das amostras colhidas na etapa de hidrólise, utilizou-se um kit enzimático (GOD-PAP) pronto para uso. Em eppendorfs, foram adicionados $10 \mu \mathrm{L}$ de amostra a $1 \mathrm{~mL}$ do reagente. A mistura foi aquecida em banho termostático a $37{ }^{\circ} \mathrm{C}$ durante 10 minutos, para que houvesse a completa reação de oxidação enzimática da glicose. Em seguida, as absorbâncias foram medidas em espectrofotômetro a 505 nm (modelo 2000, Pharmacia Biotech). Para a construção da curva de calibração, foi preparada uma solução padrão de glicose de $2,00 \mathrm{~g} \cdot \mathrm{L}^{-1}$ e a partir daí diferentes diluições de glicose foram feitas: 0,$25 ; 0,50 ; 1,00$ e $2,00 \mathrm{~g} \cdot \mathrm{L}^{-1}$. A partir da equação da reta ajustada, calculou-se a concentração de glicose em cada amostra.

\section{RESULTADOS E DISCUSSÃO}

\subsection{Caracterização química da palha in natura e pré-tratada}

Inicialmente foi feita a caracterização química da palha in natura. Em seguida, amostras de palha foram submetidas ao pré-tratamento hidrotérmico a $195{ }^{\circ} \mathrm{C} / 10$ minutos e $200 \mathrm{rpm}$, a fim de remover lignina e, principalmente, hemicelulose. Os valores obtidos na caracterização química da palha in natura e pré-tratada hidrotermicamente estão apresentados na Tabela 1, bem como a porcentagem de remoção de cada um dos componentes das amostras de palha.

Os resultados apresentados na Tabela 1 indicam que o pré-tratamento foi eficiente na remoção da hemicelulose. A composição química da palha in natura foi similar à apresentada por Barros et al., 2013. 
Tabela 1- Composição química da palha in natura e pré-tratada.

\begin{tabular}{cccc}
\hline \hline Componente (\%) & In natura & $\begin{array}{c}\text { Pré-tratada } \\
(\mathbf{1 9 5} \mathbf{C} / \mathbf{1 0} \text { min e 200 } \mathbf{~ p m ) ~}\end{array}$ & Remoção (\%) \\
\hline Celulose & 36,70 & 58,04 & 20,14 \\
Hemicelulose & 29,88 & 8,23 & 86,09 \\
Lignina & 19,74 & 24,98 & 36,10 \\
Cinzas & 6,15 & 5,54 & 54,51 \\
Extrativos & 6,24 & - & 100,00 \\
Total & 99,71 & 96,80 & - \\
Rendimento & - & 50,50 & - \\
Fator de & - & 4,17 & - \\
severidade & & \multicolumn{2}{c}{} \\
\hline \hline
\end{tabular}

* Estimado segundo Overend et al.(1987)

\subsection{Influência da agitação na velocidade de formação de glicose}

Um estudo detalhado da influência da velocidade de agitação na cinética da hidrólise enzimática deve anteceder de todas as outras variáveis operacionais, a fim de determinar a mínima velocidade de agitação que conduz a uma alta velocidade de difusão, evitando que o processo seja limitado pelos efeitos difusionais externos. Estudos realizados por Carvalho et al. (2013) mostraram que, velocidades acima de $150 \mathrm{rpm}$ resultaram em velocidades iniciais de reação praticamente iguais para a hidrólise do bagaço de cana-de-açúcar em concentração de substrato de $9,1 \%\left(\mathrm{~m}_{\text {celulose }} \cdot \mathrm{m}_{\text {total }}{ }^{-1}\right)$.

Na Tabela 2 encontram-se os valores de velocidades iniciais de reação de hidrólise para uma carga de $10 \%$ de sólidos $(\mathrm{m} / \mathrm{v})$ em agitações de $0,50,150,200,250$ e $300 \mathrm{rpm}$. As velocidades iniciais foram determinadas a partir do coeficiente angular da reta ajustada aos dados experimentais de concentração de glicose em função do tempo reacional, considerando somente a região linear da formação de glicose, ou seja, até $10 \%$ de conversão do substrato.

Tabela 2- Velocidades iniciais de hidrólise enzimática da palha da cana-de-açúcar para diferentes agitações.

\begin{tabular}{cc}
\hline \hline Agitação $(\mathbf{r p m})$ & $\begin{array}{c}\text { Velocidades iniciais } \\
\left(\text { gglicose } \mathbf{L}_{\text {solução }}{ }^{-1} \cdot \mathbf{m i n}^{-1}\right)\end{array}$ \\
\hline 0 & $0,023 \pm 1,0 \cdot 10^{-3}$ \\
50 & $0,030 \pm 2,1 \cdot 10^{-3}$ \\
150 & $0,042 \pm 2,3 \cdot 10^{-3}$ \\
200 & $0,052 \pm 1,0 \cdot 10^{-3}$ \\
250 & $0,053 \pm 5,8 \cdot 10^{-4}$ \\
300 & $0,055 \pm 2,6 \cdot 10^{-3}$ \\
\hline \hline
\end{tabular}




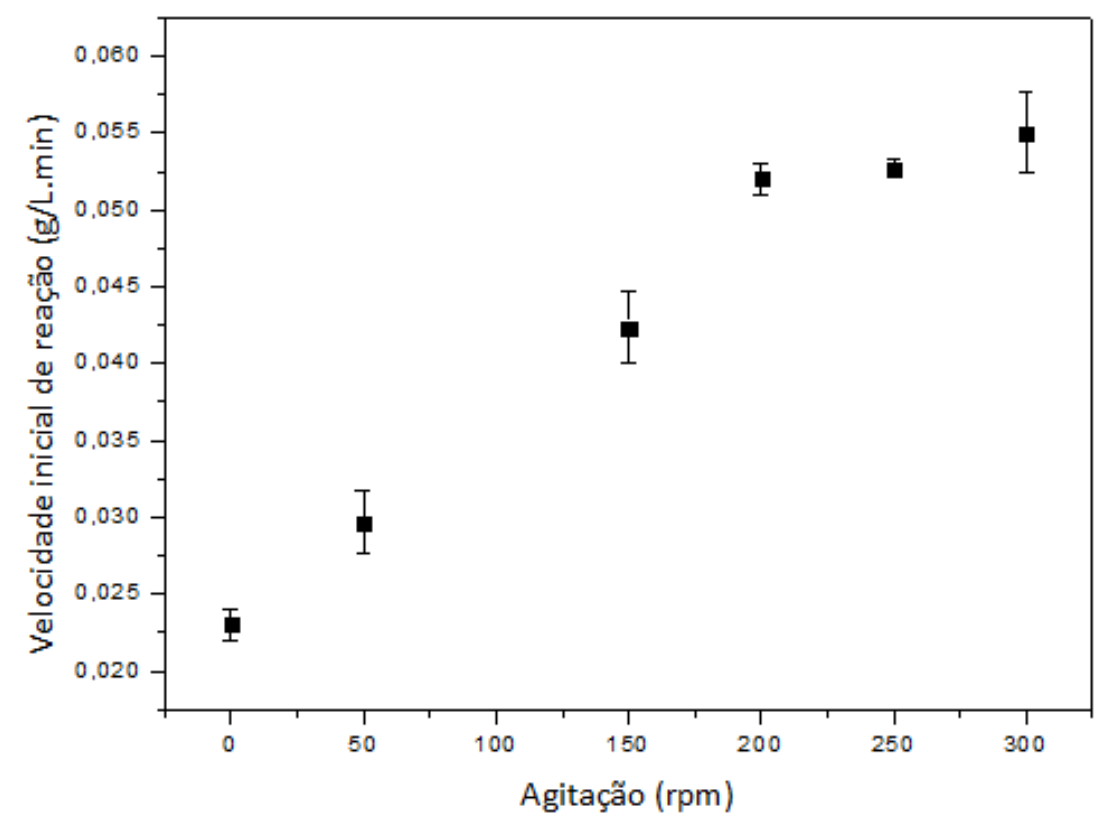

Figura 1- Velocidades iniciais de reação em função da velocidade de agitação, para concentração de substrato de $10 \%$ de sólidos, em $50{ }^{\circ} \mathrm{C}, \mathrm{pH} 5$ e concentração de enzima de $5 \mathrm{FPU} \cdot \mathrm{g}_{\text {celulose }}{ }^{-1}$.

Através da Figura 1, observa-se que houve um aumento significativo na velocidade de reação com o aumento de agitação até $200 \mathrm{rpm}$, variando de 0,023 a $0,052 \mathrm{~g}_{\text {glicose }} \cdot \mathrm{L}_{\text {solução }}{ }^{-}$ ${ }^{1} \cdot \mathrm{min}^{-1}$, indicando que havia resistência ao transporte de massa externo, o qual foi minimizado com o aumento da velocidade de agitação. Agitações acima de $200 \mathrm{rpm}$ resultaram em velocidades de reação praticamente invariantes, podendo considerar a hipótese de que as limitações difusionais externas são desprezíveis face ao fenômeno de adsorção e reação na superfície do substrato, e que a partir dessa velocidade de agitação atingiu-se a velocidade máxima de reação $\left(\mathrm{V}_{\text {máx }}\right)$. Desta forma, com o objetivo de minimizar gasto energético, efeitos difusionais externos e ocorrências de inativação da enzima devido ao cisalhamento, se escolheu $200 \mathrm{rpm}$ como velocidade ótima de agitação para dar sequência ao estudo cinético da hidrólise.

\subsection{Influência da concentração de substrato}

Para vários sistemas enzimáticos, a velocidade inicial varia hiperbolicamente com a concentração do substrato. No caso da hidrólise enzimática de palha de cana-de-açúcar por Cellic ${ }^{\circledR}$ CTec2, o comportamento observado foi o mesmo, conforme mostrado na Figura 2. A expressão matemática que relaciona valores de velocidades iniciais e a concentração de substrato é mostrada na Equação 1, conhecida como equação de Michaelis-Menten:

$$
V=\frac{V_{\text {máx }} \cdot S}{K_{m}+S}
$$

onde: $\mathrm{V}$ é a velocidade de consumo de substrato em $\mathrm{g}_{\text {glicose }} \cdot \mathrm{L}_{\text {solução }}{ }^{-1} \cdot \mathrm{min}^{-1}, \mathrm{~V}_{\text {máx }}$ é a velocidade máxima de consumo do substrato em $\mathrm{g}_{\text {glicose }} \cdot \mathrm{L}_{\text {solução }}{ }^{-1} \cdot \min ^{-1} ; \mathrm{S}$ é a concentração de substrato em $g_{\text {glicose potencial }} L_{\text {solução }}{ }^{-1}$ e $K_{m}$ a constante de Michaelis-Menten em $g_{\text {glicose potencial }} L_{\text {solução }}{ }^{-1}$. 
Experimentos com palha de cana-de-açúcar foram realizados a fim de estudar o efeito da concentração de substrato na velocidade de reação enzimática. A concentração de palha adicionada em cada ensaio experimental variou de $2,5 \%$ a $10 \%$ (massa de palha seca/volume reacional), correspondendo a uma concentração de glicose potencial de 16,12 a 64,48 gglicose potencial ${ } L_{\text {solução }}{ }^{-1}$. A concentração volumétrica de enzima adicionada em todos os ensaios foi de $284 \mathrm{FPU} \cdot \mathrm{L}_{\text {solução }}{ }^{-1}$, sendo a atividade enzimática da Cellic ${ }^{\circledR} \mathrm{CTec} 2=225 \mathrm{FPU} \cdot \mathrm{mL}_{\text {solução }}{ }^{-1}$.

Na Tabela 3 encontram-se os valores de velocidades iniciais de reação de hidrólise para diferentes concentrações de substrato.

Tabela 3- Velocidades iniciais de hidrólise enzimática da palha da cana-de-açúcar para diferentes concentrações de substrato.

\begin{tabular}{cc}
\hline $\begin{array}{c}\text { Concentração de } \\
\text { substrato }(\mathbf{m} / \mathbf{v})\end{array}$ & $\begin{array}{c}\text { Velocidades iniciais } \\
\left(\mathbf{g}_{\text {glicose }} \mathbf{L}_{\text {solução }} \mathbf{- 1}^{-1} \cdot \mathbf{m i n}^{-1}\right)\end{array}$ \\
\hline 2,5 & $0,0317 \pm 2,52 \cdot 10^{-3}$ \\
5,0 & $0,0403 \pm 3,21 \cdot 10^{-3}$ \\
7,5 & $0,0400 \pm 5,77 \cdot 10^{-4}$ \\
10,0 & $0,0520 \pm 1,00 \cdot 10^{-3}$ \\
\hline \hline
\end{tabular}

Na Figura 2 foram plotados os valores de velocidades iniciais de reação em função da concentração de substrato.

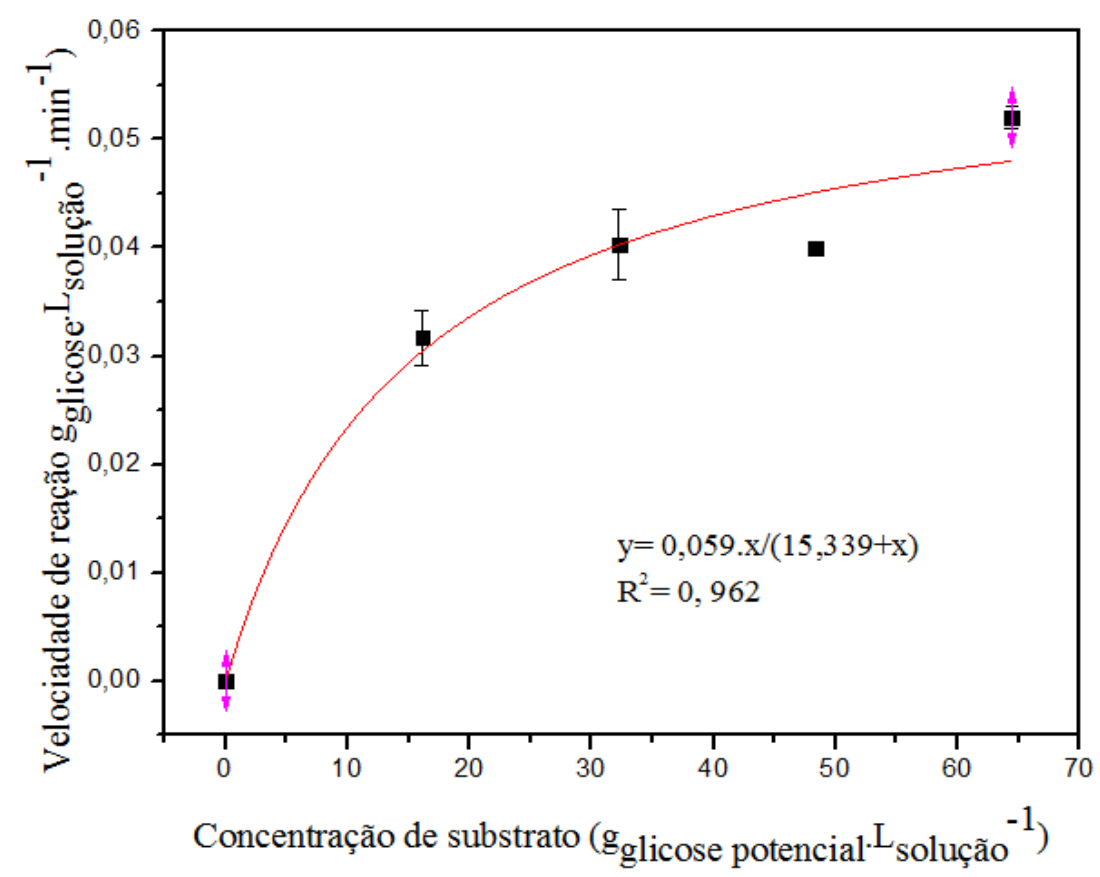

Figura 2- Velocidades iniciais de reação em função da concentração de substrato para concentração volumétrica de atividade enzimática de $284 \mathrm{FPU} \cdot \mathrm{L}_{\text {solução }}{ }^{-1}, 50{ }^{\circ} \mathrm{C}$ e pH 5.

Para determinar os parâmetros cinéticos de Michaelis-Menten, $\mathrm{V}_{\text {máx }}$ e $\mathrm{K}_{\mathrm{m}}$, utilizou-se a ferramenta de ajuste não-linear do software OriginPro 8.0. Os valores dos parâmetros cinéticos obtidos foram: $\mathrm{V}_{\text {máx }}=0,059 \mathrm{~g}_{\text {glicose }} \cdot \mathrm{FPU}^{-1} \cdot \mathrm{min}^{-1}$ e $\mathrm{K}_{\mathrm{m}}=15,339 \mathrm{~g}_{\text {glicose potencial }} \cdot \mathrm{L}_{\text {solução }}{ }^{-1}$. 
$\mathrm{O}$ valor encontrado de $\mathrm{V}_{\text {máx }}$ condiz com os valores de velocidades iniciais para agitações acima de $200 \mathrm{rpm}$ (Tabela 2), indicando o modelo de Michaelis-Menten se ajustou bem aos dados experimentais quando concentrações de produto ainda são baixas. Além disso, observase, a partir da Figura 2, que para concentrações baixas de substratos, até aproximadamente 16 $\mathrm{g}_{\text {glicose potencial }} \mathrm{L}_{\text {solução }}{ }^{-1}$, a velocidade de reação varia quase que linearmente com o aumento de concentração de substrato. Já para concentrações maiores de substrato a velocidade aumenta por incrementos menores em resposta ao aumento da concentração de substrato, até que se alcança um ponto acima do qual o aumento de velocidade é insignificante, sendo esse ponto chamado de velocidade máxima $\left(\mathrm{V}_{\text {máx }}=\mathrm{k} \cdot \mathrm{E}\right)$.

A partir de $\mathrm{V}_{\text {máx }}$ é possível calcular a constante de velocidade $\mathrm{k}$, sendo a concentração volumétrica de atividade enzimática $=284 \mathrm{FPU} \cdot \mathrm{L}_{\text {solução }}{ }^{-1}$, o valor encontrado foi $\mathrm{k}=2,11 \cdot 10^{-4}$ $\mathrm{g}_{\text {glicose }} \cdot \mathrm{FPU}^{-1} \cdot \mathrm{min}^{-1}$.

\section{CONSIDERAÇÕES FINAIS}

Neste trabalho foi realizado um estudo cinético da hidrólise enzimática da palha da cana-de-açúcar através do qual pôde-se avaliar a influência da agitação e concentração de substrato na velocidade da reação a fim de determinar os parâmetros cinéticos da hidrólise enzimática. Os dados experimentais obtidos mostraram que, na faixa 0 a $200 \mathrm{rpm}$, ocorreu sempre um aumento na velocidade de reação de hidrólise, indicando que havia resistência ao transporte de massa externo, o qual foi minimizado com o aumento da velocidade de agitação. A partir de $200 \mathrm{rpm}$, a velocidade de reação foi praticamente invariante, sendo esta selecionada como velocidade ótima de agitação. Foi possível ajustar o modelo de MichaelisMenten aos dados experimentais de velocidades iniciais da reação e os valores dos parâmetros cinéticos foram: $\mathrm{V}_{\text {máx }}=0,059 \mathrm{~g}_{\text {glicose }} \cdot \mathrm{L}_{\text {solução }}{ }^{-1} \cdot \min ^{-1}, \quad \mathrm{k}=2,11.10^{-4} \mathrm{~g}_{\text {glicose }} \cdot \mathrm{FPU}^{-1} \cdot \mathrm{min}^{-1} \mathrm{e}$ $\mathrm{K}_{\mathrm{m}}=15,339 \mathrm{~g}_{\text {glicose potencial }} \mathrm{L}_{\text {solução }}{ }^{-1}$. Para trabalhos posteriores, estudar-se-á o efeito de inibição de produto ajustando modelos cinéticos de inibição em experimentos de longa duração, ou seja, para altas conversões de substrato.

\section{AGRADECIMENTOS}

Os autores agradecem ao Programa de Recursos Humanos da Agência Nacional de Petróleo, Gás e Biocombustíveis (PRH 44-ANP), à CAPES e ao CNPq pelo apoio financeiro.

\section{REFERÊNCIAS BIBLIOGRÁFICAS}

BARROS, R.R.O.; PAREDES, R.S.; ENDO, T.; BON, E.P.S.; LEE, S. Association of wet disk milling and ozonolysis as pretreatment for enzymatic saccharification of sugarcane bagasse and straw. Bioresour. Technol., v. 136, p. 288-294, 2013.

BINOD, P.; JANU, K.U.; SINDHU, R.; PANDEY, A. Hydrolysis of lignocellulosic biomass for bioethanol production. Biofuels., Burlington: Academic Press, p. 229-250, 2011.

CARVALHO, M.L.; SOUZA, R.JR.; RODRÍGUEZ-ZÚÑIGA, U.F.; SUAREZ, C.A.G.; RODRIGUES, D.S.; GIORDANO, R.C.; GIORDANO, R.L.C. Hydrolysis of sugarcane bagasse. Braz. J. Chem. Eng., v. 30, n. 03, p. 437-447, 2013. 
GAN, Q.; ALLEN, S.J.; TAYLOR, G. Kinetic dynamics in heterogeneous enzymatic hydrolysis of cellulose: an overview, an experimental study and mathematical modeling. Process Biochem., v. 38, p. 1003-1018, 2003.

GOUVEIA, E.R.; NASCIMENTO, R.J.; SOUTO-MAIOR, A.M.; ROCHA, G.J.M. Validação da metodologia para caracterização química do bagaço de cana-de-açúcar. Quim. Nova., v. 32, n. 6, p. 1500-1503, 2009.

KOOTSTRA, A.M.J.; MOSIER, N.S.; SCOTT, E.L.; BEEFTINK, H.H.; SANDERS, J.P.M. Differential effects of mineral and organic acids on the kinetics of arabinose degradation under lignocellulose pretreatment conditions. Biochem. Eng. J., v. 43, p. 92-97, 2009.

LEAL, M.L.R.V.; GALDOS, M.V.; SCARPARE, F.V.; SEABRA, J.E.A.; WALTER, A.; OLIVEIRA, C.O.F. Sugarcane straw availability, quality, recovery and energy use: A literature review. Biomass Bioenergy., v. 53, p. 11-19, 2013.

MAEDA, R.N.; SERPA, V.I.; ROCHA, V.A.L.; MESQUITA, R.A.A.; ANNA, L.M.M.S.; CASTRO, A.M.; DRIEMEIER, C.E.; PEREIRA JR., N.; POLIKARPOV, I. Enzymatic hydrolysis of pretreated sugar cane bagasse using Penicillium funiculosum and Trichoderma harzianum cellulases. Process Biochem., v. 46, n. 5, p. 1196-1201, 2011.

OVEREND, R.P.; CHORNET, E.; GASCOIGNE, J. A. Fractionation of lignocellulosics by steam-aqueous pretreatments. Philosophical Transaction of the Royal Society A,v. 321(1561):523-536, 1987.

ROCHA, G.J.M.; SILVA, F.T.; CURVELO, A.A.S.; ARAÚJO, G.T. A fast and accurate method for determination of cellulose and polyoses by HPLC. Fifth Braz. Symp. Chem., 1997.

SANTOS, F.A.; QUEIRÓZ, J.H.; COLODETTE, J.L.; FERNANDES, S.A. GUIMARÃES, V.M.; REZENDE, S.T. Potencial da palha da cana-de-açúcar para a produção de etanol. Quim. Nova., v. 35, p. 1004-1010, 2012.

SARKAR, N; GHOSH, S.K.; BANNERJEE, S.; AIKAT, K. Bioethanol production from agricultural wastes: An overview. Renew. Energy., v. 37, p. 19-27, 2012. 